\title{
Yuzu (Citrus junos Tanaka) Peel Attenuates Dextran Sulfate Sodium-induced Murine Experimental Colitis
}

Hiroko Abe ${ }^{1 *}$, Mika Ishioka², Yasuko Fujita ${ }^{1}$, Aya Umeno', Mayu Yasunaga ${ }^{1}$, Akihiko Sato ${ }^{3}$, Shigehiko Ohnishi ${ }^{3}$, Shingo Suzuki ${ }^{4}$, Noriko Ishida ${ }^{5}$, Mototada Shichiri ${ }^{5}$, Yasukazu Yoshida ${ }^{1}$ and Yoshihiro Nakajima ${ }^{1}$

\footnotetext{
${ }^{1}$ Health Research Institute, National Institute of Advanced Industrial Science and Technology (AIST), 2217-14 Hayashi-cho, Takamatsu, Kagawa 761-0395, JAPAN

${ }^{2}$ Shikoku Research Institute Inc., 2109-8 Yashimanishi, Takamatsu, Kagawa 761-0113, JAPAN

${ }^{3}$ Kagawa Prefectural Industrial Technology Center, 587-1 Goutou, Takamatsu, Kagawa 761-8031, JAPAN

${ }^{4}$ Faculty of Medicine, Kagawa University, 1750-1 Miki-cho, Kagawa 761-0793, JAPAN

${ }^{5}$ Biomedical Research Institute, National Institute of Advanced Industrial Science and Technology (AIST), 1-8-31 Midorigaoka, Ikeda, Osaka 563-8577, JAPAN
}

\begin{abstract}
Ulcerative colitis is a well-known inflammatory bowel disease. Although there are drugs that are effective against this disease, the prevention and attenuation of ulcerative colitis by food rich in functional ingredients without side effects is desired because some drugs have side effects. In this study, we investigated the effects of yuzu (Citrus junos Tanaka), a citrus fruit native to northeast Asia, on a mouse dextran sulfate sodium (DSS)-induced colitis model. Mice given drinking water containing DSS showed significant weight loss, colon shortening, diarrhea, and visible fecal blood. In contrast, mice fed a diet containing $5 \%$ yuzu peel for $14 \mathrm{~d}$ before receiving DSS showed significant attenuation of these phenotypes. To clarify the mechanism underlying the attenuation, we investigated the anti-inflammatory and antioxidant effects of yuzu peel. We found that yuzu peel extract suppressed tumor necrosis factor- $\alpha(\mathrm{TNF}-\alpha)$ production in lipopolysaccharide

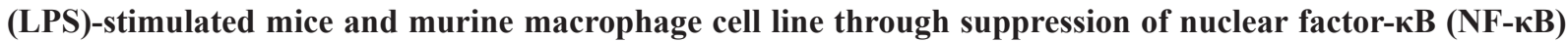
activation. In addition, we confirmed that yuzu peel extract had a moderate antioxidant effect. These results suggest that yuzu peel attenuates the pathologies of DSS-induced colitis by coordinately suppressing inflammation and oxidative stress against lipids in vivo.
\end{abstract}

Key words: yuzu, experimental colitis, inflammation, oxidative stress, antioxidant against lipids, citrus flavonoid

\section{Introduction}

Ulcerative colitis is an idiopathic inflammatory bowel disease characterized by intestinal inflammation, and the number of patients is increasing in developed countries ${ }^{1)}$. It has been reported that patients with active ulcerative colitis status have high activated macrophage, monocyte, and neutrophil counts in peripheral blood or inflamed gut ${ }^{2,3)}$. The activated macrophages and monocytes generate proinflammatory cytokines, including tumor necrosis factor- $\alpha$ $(\mathrm{TNF}-\alpha)^{4,5)}$, which induce inflammation in the intestines ${ }^{6}$. Reactive oxygen species generated by the activated macrophages and neutrophils also contribute to intestinal oxidative injury in ulcerative colitis patients ${ }^{7)}$. Thus, it has been believed that both inflammation and oxidative stress are involved in the development and progress of ulcerative colitis $^{8)}$.

Such drugs as aminosalicylates and corticosteroids are currently used for the treatment of this disease. However, they have serious side effects, including allergic reaction ${ }^{9,10)}$. It is therefore ideal that the pathogenesis of ulcerative colitis be prevented or attenuated by the ingestion of food without side effects.

Yuzu(Citrus junos Tanaka) is a popular citrus fruit native to northeast Asia, including Japan, China, and Korea $^{11)}$. Yuzu has been used in traditional Chinese medi-

Abbreviations: DSS; dextran sulfate sodium, HPLC; high performance liquid chromatography, IL; interleukin, LPS; lipopolysaccharide, MS; mass spectrometry, NF- $\mathrm{BB}$; nuclear factor- $\kappa \mathrm{B}$, PBS; phosphate-buffered saline, TNF- $\alpha$; tumor necrosis factor- $\alpha$

\footnotetext{
*Correspondence to: Hiroko Abe, Health Research Institute, AIST, 2217-14 Hayashi-cho, Takamatsu, Kagawa 761-0395, JAPAN

E-mail: abe-abe@aist.go.jp

Accepted October 19, 2017 (received for review August 17, 2017)

Journal of Oleo Science ISSN 1345-8957 print / ISSN 1347-3352 online

http://www.jstage.jst.go.jp/browse/jos/ http://mc.manusriptcentral.com/jjocs
} 
cine $^{12)}$, beverages, cuisines, and $\operatorname{cosmetics}^{13,14)}$. It has been reported that yuzu contains many bioactive compounds that show anti-inflammatory and/or antioxidant activities, including ascorbic acid, $\beta$-carotene, flavonoids, and limonoids ${ }^{15}$. The extract of yuzu inhibits platelet aggregation, prevents myocardial infarction-induced ventricular dysfunction and structural remodeling of myocardium, and exerts an antidiabetic effect ${ }^{11,16,17}$. Although yuzu has many health benefits, there is no report of its beneficial effects on ulcerative colitis.

In this study, we examined the effect of yuzu on ulcerative colitis induced by dextran sulfate sodium(DSS) in mouse. We found that yuzu peel significantly attenuated DSS-induced colitis symptoms due to the coordination between its anti-inflammatory and antioxidant effects.

\section{Experimental}

\subsection{Extraction of yuzu peel and preparation of samples}

Yuzu peel paste YP-01, which consists of yuzu peel after squeezing out the juice, was obtained from Shikoku Research Institute Inc. (Kagawa, Japan). The yuzu peel paste was stored at $-80^{\circ} \mathrm{C}$. For the extraction of the aqueous fraction, $10 \mathrm{~g}$ of frozen yuzu peel paste and $10 \mathrm{~mL}$ of cold serum-free RPMI 1640 medium (Sigma-Aldrich, MO) supplemented with $2 \mathrm{mM}$ L-glutamine or cold phosphate-buffered saline (PBS) were transferred to a $50 \mathrm{~mL}$ conical centrifuge tube. The mixture was vortexed vigorously on the highest setting for 30 seconds to produce a suspension of yuzu peel paste. The suspension was further mixed in a rotator (RT-5; TAITEC Corporation., Saitama, Japan) at 2.5 $\mathrm{r} / \mathrm{min}$ for $15 \mathrm{~min}$ at $5^{\circ} \mathrm{C}$, and then centrifuged at $1100 \mathrm{x} \mathrm{g}$ for $5 \mathrm{~min}$ at $5^{\circ} \mathrm{C}$. The resulting supernatant was used as $50 \%$ yuzu peel extract.

\subsection{Quantitative analysis of flavonoids and ascorbic acid}

The 50\% yuzu peel extract extracted with RPMI 1640 medium (hereinafter, RPMI 1640 extract of yuzu peel)was diluted 5-fold with $\mathrm{DMSO} / \mathrm{MeOH}(1: 1 \mathrm{v} / \mathrm{v})$ and then measured in a UFLC system (Prominence UFLC; Shimadzu Corporation, Kyoto, Japan) equipped with a Shim-pack XR-ODS column (Shimadzu Corporation) maintained at $30^{\circ} \mathrm{C}$ at the flow rate of $0.6 \mathrm{~mL} / \mathrm{min}$. The absorbance at 280 $\mathrm{nm}$ was monitored. Formic acid $(0.1 \%(\mathrm{v} / \mathrm{v}) ; \mathrm{A})$ and $99.9 \%$ acetonitrile containing $0.1 \%$ formic acid (B) were used as the gradient elution system. The mobile phase was $95 \% \mathrm{~A}$, which was decreased to $50 \%$ over a period of $18.7 \mathrm{~min}$. It was further decreased to $5 \% \mathrm{~A}$ in $0.5 \mathrm{~min}$ and maintained at that concentration for another $1.5 \mathrm{~min}$. Hesperidin (Wako Pure Industries, Ltd., Osaka, Japan) and naringin (SigmaAldrich)were dissolved in DMSO/MeOH $(1: 1 \mathrm{v} / \mathrm{v})$ and diluted to prepare standard solutions $(8 \mu \mathrm{M}$ to $324 \mu \mathrm{M})$ for the creation of a calibration curve for each chemical. Each flavonoid was quantified on the basis of the calibration curve of hesperidin or naringin.

Ascorbic acid content in the yuzu peel extract extracted with PBS (hereinafter, PBS extract of yuzu peel) was determined with an Ascorbic Acid Colorimetric/Fluorometric Assay Kit(BioVision, CA) in accordance with the manufacturer's instructions.

\subsection{Animals}

Male BALB/c mice were purchased from Clea Japan Inc. (Tokyo, Japan). The animals were fed a standard laboratory diet (CE-2; Clea Japan Inc.) and water ad libitum. The animal room was maintained at $23 \pm 2^{\circ} \mathrm{C}$ and $50 \pm 10 \% \mathrm{hu}-$ midity under a $12 \mathrm{~h}$ light (8:00-20:00) and dark (20:008:00) cycle. The animals were acclimated to the laboratory environment for at least one week before the experiment. All the experimental procedures were approved by the Institutional Animal Care and Use Committee of the National Institute of Advanced Industrial Science and Technology.

\subsection{Mouse DSS-colitis model}

Mice were randomly divided into four experimental groups: two groups (5 animals each) were fed a diet containing $50 \mathrm{~g} / \mathrm{kg}(\mathrm{w} / \mathrm{w})$ yuzu peel paste daily for $14 \mathrm{~d}$. The remaining two groups were fed a standard diet. One group fed yuzu peel paste was given drinking water containing $4 \%(\mathrm{w} / \mathrm{v})$ DSS for $5 \mathrm{~d}$ to induce colitis while yuzu peel paste feeding was continued. One group fed the standard diet was also given drinking water containing $4 \%(\mathrm{w} / \mathrm{v})$ DSS for $5 \mathrm{~d}$. To determine the severity of clinical colitis, body weight, stool consistency, and visible fecal blood were evaluated daily. Stool consistency and visible fecal blood were scored as described by Melgar et al. ${ }^{18)}$ with slight modifications, as shown in Table 1.

\subsection{LPS-induced inflammation in vivo and measurement of plasma cytokines}

Male BALB/c mice (6 weeks old, 18 to $22 \mathrm{~g}$ ) without anesthesia were intraperitoneally administered $13 \mu \mathrm{g}$ of lipopolysaccharide (LPS) with or without PBS extract of yuzu peel at various concentrations $(200 \mu \mathrm{L} /$ cavity $)$. A mixture of LPS and yuzu peel extract for intraperitoneal administration was prepared by mixing in PBS. A 26-gauge, 1/2inch injection needle (Terumo Co., Tokyo, Japan) and a 1 $\mathrm{mL}$ syringe(Terumo Co., Tokyo, Japan) were used for each

Table 1 Scores of diarrhea and visible fecal blood.

\begin{tabular}{ccc}
\hline Score & Diarrhea Score & Visible Fecal Blood \\
\hline 0 & Normal pellets & Normal \\
1 & Slightly loose feces & Slightly bloody \\
2 & Loose feces & Adherence of blood around anus \\
3 & Watery diarrhea & Bleeding through the anus \\
\hline
\end{tabular}


mouse. Peripheral blood of individual mice was collected from the retro-orbital sinus. ELISA kits were used for measuring plasma cytokines, namely, TNF- $\alpha$ (Biosource Europe S. A., Nivelles, Belgium), interleukin (IL)-12 (total), and interferon- $\gamma($ IFN- $\gamma)$ (Thermo Fisher Scientific Inc., MA) in accord-ance with the manufacturer's instructions.

\subsection{Cells and cell culture}

The murine macrophage-like cell line RAW 264.7(DS Pharma Biomedical Co., Osaka, Japan) and RAW 264.7 cells expressing secreted embryonic alkaline phosphatase (SEAP) (Raw-Blue cells, InvivoGen, CA) were cultured in RPMI 1640 medium (Sigma-Aldrich) supplemented with $10 \%$ fetal bovine serum, $2 \mathrm{mM}$ L-glutamine, $100 \mathrm{U} / \mathrm{mL}$ penicillin, and $100 \mu \mathrm{g} / \mathrm{mL}$ streptomycin, and were maintained at $37^{\circ} \mathrm{C}$ in a humidified atmosphere of $5 \% \mathrm{CO}_{2}$.

\subsection{Measurement of TNF- $\alpha$ produced by RAW 264.7}

RAW 264.7 cells $\left(1 \times 10^{5}\right.$ cells/well $)$ were plated into 96-well plates for $4 \mathrm{~h}$. The cells were treated with RPMI 1640 extract of yuzu peel at the concentration of 0 to $1 \%$ with or without LPS (250 ng/mL), and incubation was carried out for $24 \mathrm{~h}$.

\subsection{SEAP reporter assay}

Raw-Blue cells $\left(1 \times 10^{5}\right.$ cells/well $)$ were plated into 96-well plates and treated with RPMI 1640 extract of yuzu peel at the concentration of 0 to $1 \%$ with LPS (50 ng/mL). Then, the cells were incubated for $24 \mathrm{~h}$. SEAP activity in the supernatants was measured using Quanti-Blue(InvivoGen).

\subsection{Indirect immunofluorescence assays and confocal microscopy}

RAW 264.7 cells $\left(2.5 \times 10^{4}\right.$ cells/well $)$ were cultured in an 8-well cell culture chamber slide(Thermo Fisher Scientific). The cells were treated with 1\% RPMI 1640 extract of yuzu peel for $24 \mathrm{~h}$ at $37^{\circ} \mathrm{C}$, and then treated with LPS (50 $\mathrm{ng} / \mathrm{mL}$ ) for $30 \mathrm{~min}$ at $37^{\circ} \mathrm{C}$. Subcellular localization of nuclear factor- $\kappa \mathrm{B}(\mathrm{NF}-\kappa \mathrm{B})$ p65 was examined by indirect immunofluorescence assay as reporter previously ${ }^{19)}$ using a rabbit polyclonal antibody against p65 (1:500, Cell Signaling Technology Inc., MA) and an Alexa Fluor 546-conjugated goat anti-rabbit antibody (1:500, Thermo Fisher Scientific).

Nuclear counterstaining was performed with TO-PRO-3 (1:10000, Thermo Fisher Scientific). The cells were imaged under a confocal laser scanning microscope(LSM 700, Carl Zeiss, Oberkochen, Germany). Fluorescence intensity in the cytoplasm was determined from the p65 fluorescence intensity in the $\mathrm{TO}-\mathrm{PRO}-3$-negative region divided by the p65 fluorescence intensity in the entire region including the TO-PRO-3-positive region.

\subsection{Measurement of lipid peroxidation}

The antioxidant activity of PBS extract of yuzu peel was determined by measuring oxygen uptake as described previously $^{20)}$. Briefly, micelles were prepared by vigorously vortexing methyl linoleate $(0.2 \%(\mathrm{v} / \mathrm{v}))$ in PBS containing $0.05 \mathrm{M}$ cholic acid. Free-radical-mediated oxidation of methyl linoleate generates conjugated diene hydroperoxides together with the consumption of oxygen via a straightforward mechanism. The oxidation of methyl linoleate and its inhibition by antioxidants were monitored by oxygen uptake measurement using an oxygen monitor equipped with a Clark-type oxygen electrode (YSI model $5300, \mathrm{OH}$ ) at $37^{\circ} \mathrm{C}$. Hydrophilic 2,2'-azobis (2-amidinopropane) dihydrochloride was used as an azo initiator.

\subsection{Statistical analysis}

Data are expressed as means \pm standard error (SE). Statistical analyses were performed using Prism version 5.0c (GraphPad Software, CA). The statistical significance of any difference in each parameter among the groups was evaluated by one-way analysis of variance (ANOVA) and Dunnett's test as the post hoc test. $p$ Values $<0.05$ were considered statistically significant.

\section{Results and discussion}

3.1 Characterization of bioactive components in yuzu peel

First, we analyzed the bioactive components in yuzu peel used in this study. RPMI 1640 extract of yuzu peel was subjected to HPLC and HPLC-MS/MS. The results showed that narirutin $(774 \pm 12 \mu \mathrm{M})$, hesperetin 7-(2,6-dirhamnosylglucoside $)(356 \pm 5 \mu \mathrm{M})$, naringin $(513 \pm 10 \mu \mathrm{M})$, hesper$\operatorname{idin}(1118 \pm 15 \mu \mathrm{M})$, neohesperidin $(635 \pm 9 \mu \mathrm{M})$, and rhamnetin 3-o-rutinoside (concentration not determined) were the major compounds (Table 2, Fig. S1). The bioactive components and their concentration in PBS extract of yuzu peel were almost the same as those in the RPMI 1640 extract of yuzu peel(data not shown). We also identified ascorbic acid $(602 \pm 68 \mu \mathrm{M})$ in the PBS extract of yuzu peel. These results suggest the possibility of yuzu peel having an attenuating effect on ulcerative colitis because the bioactive components identified in yuzu peel exhibit both anti-

Table 2 Flavonoid concentrations in yuzu peel extract.

\begin{tabular}{lc}
\hline \multicolumn{1}{c}{ Compound } & Concentration $(\mu \mathrm{M})$ \\
\hline Narirutin & $773.9 \pm 11.8$ \\
Hesperetin7-(2,6-dirhamnosylglucoside) & $355.8 \pm 4.5$ \\
Naringin & $512.6 \pm 10.4$ \\
Hesperidin & $1117.9 \pm 14.9$ \\
Neohesperidin & $635.2 \pm 9.2$ \\
\hline
\end{tabular}

Means and standard deviations of concentrations of four replicates. 
inflammatory and antioxidant effects.

\subsection{Attenuating effect of yuzu peel in mouse DSS-in- duced colitis model}

Next, we examined whether yuzu peel attenuates DSSinduced colitis in a mouse model. Mice were fed powder diet containing $50 \mathrm{~g} / \mathrm{kg}$ yuzu peel paste for $14 \mathrm{~d}$ and then given drinking water containing $4 \%(\mathrm{w} / \mathrm{v})$ DSS for $5 \mathrm{~d}$ to induce colitis while yuzu peel paste feeding was continued. Mice given DSS showed typical colitis symptoms, including weight loss (Fig. 1A, white circles), diarrhea (Fig. 1B, white circles), rectal bleeding (Fig. 1C, white circles), and colon shortening caused by edematous inflammation of the colon $^{21)}$ (Fig. 1D, yuzu - and DSS + ). Diarrhea (Fig. 1B, black circles) and visible fecal blood (Fig. 1C, black circles) were significantly attenuated in DSS-treated mice fed yuzu peel. In addition, colon shortening was also significantly suppressed (Fig. 1D, yuzu + and DSS +). We noted that TNF- $\alpha$ and IL-12 levels in the culture supernatants from a section of colon in DSS-treated mice fed yuzu peel tended

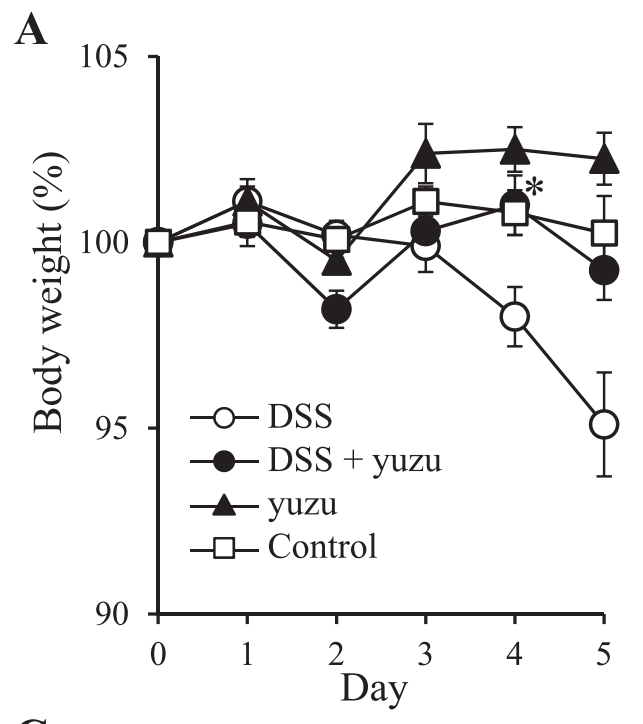

C

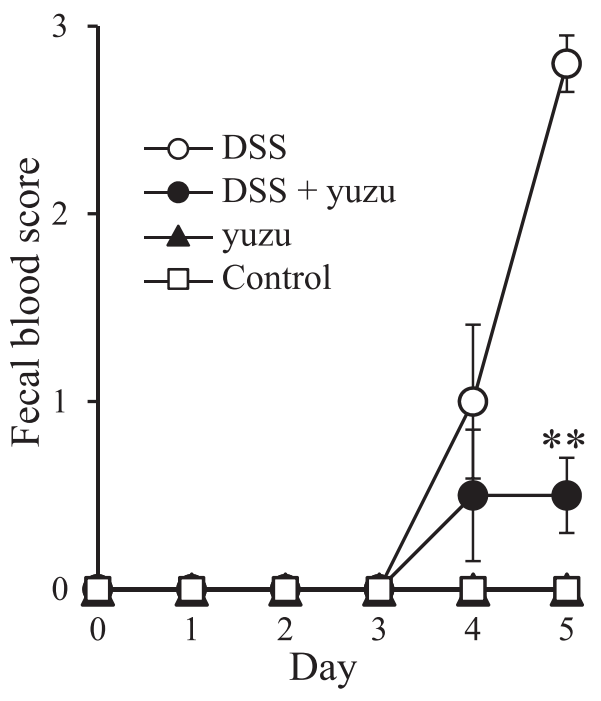

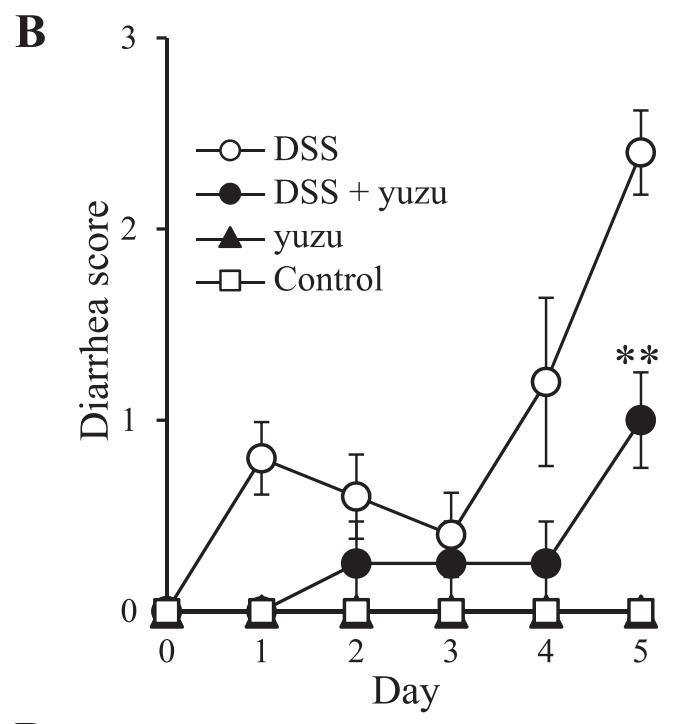

D

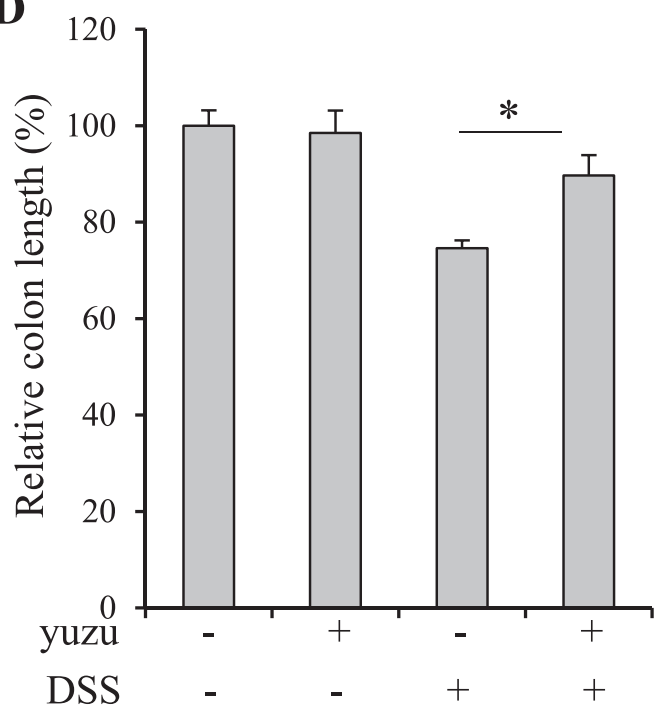

Fig. 1 Attenuating effect of yuzu peel on DSS-induced colitis. Mice were fed powder diet containing $50 \mathrm{~g} / \mathrm{kg}$ yuzu peel paste for $14 \mathrm{~d}$ and then given drinking water containing $4 \%(\mathrm{w} / \mathrm{v})$ DSS for $5 \mathrm{~d}$ to induce colitis while yuzu peel paste feeding was continued. The attenuating effect of yuzu peel was evaluated in terms of (A) body weight, (B) diarrhea score, (C) visible fecal blood score, and (D) colon length. Data are expressed as means \pm SE of five animals in each group. * $p<$ $0.05, * * p<0.01$ for comparison of DSS-treated mice fed the standard diet(DSS) with DSS-treated mice fed diet containing yuzu peel(DSS + yuzu). (A) Body weight change was calculated using body weight on day 0 as the initial body weight. (D) Relative colon length was compared with that of non-treated mice fed the standard diet (yuzu- and DSS-). 


\section{Yuzu Peel Attenuates DSS-induced Colitis}

to be lower than those in the DSS-treated mice fed the standard diet, although these differences were not statistically significant(Fig. S2). Furthermore, we examined the plasma levels of totally assessed hydroxyeicosatetraenoic acids (tHETEs), which are the lipid peroxidation products of the free and ester forms of arachidonic acids. The plasma levels of tHETEs (5-, 12-, and 15-HETEs) tended to be suppressed in mice fed yuzu peel compared to those fed the control diet with and without DSS, although the differences were not statistically significant ${ }^{22)}$ (Fig. S3). These results suggested that yuzu peel partially suppressed inflammation of the colon and oxidative stress. On the other hand, the phenotypes of non-treated mice fed diet containing yuzu peel were not significantly different from those of non-treated mice fed the standard diet(Fig. 1). Together, these results demonstrate that yuzu peel had an attenuating effect on DSS-induced colitis.

\subsection{Anti-inflammatory effect of yuzu peel}

To elucidate the mechanism underlying the attenuating effect of yuzu peel on DSS-induced colitis, we investigated the anti-inflammatory effect of yuzu peel in vivo and in vitro. In the in vivo experiment, $13 \mu \mathrm{g}$ of LPS and PBS extract of yuzu peel of different concentrations were simultaneously administered intraperitoneally into the mice. Plasma was collected at 1, 3, and $6 \mathrm{~h}$ for measurements of TNF- $\alpha$, IL-12, and IFN- $\gamma$, respectively, based on our previous finding of the peak times of the respective proinflammatory cytokines in plasma of LPS-treated mice ${ }^{23)}$. As shown in Fig. 2A, TNF- $\alpha$ level in plasma of mice injected with $0.5 \%$ yuzu peel extract was significantly reduced compared with that of mice treated with LPS alone. The levels of IL-12 and IFN- $\gamma$ also tended to decrease, but the decrease was not statistically significant (Fig. 2A). Suppression of TNF- $\alpha$ production was also observed in RAW264.7 cells (Fig. 2B) without exhibiting cytotoxicity (Fig. S4). These results demonstrated that yuzu peel has anti-inflammatory effect, as reported previously ${ }^{12,16)}$.

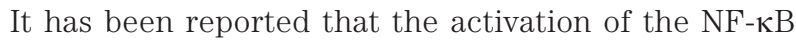
pathway, a representative proinflammatory signaling pathway $^{24)}$, following production of proinflammatory cytokines, including TNF- $\alpha$, is deeply involved in the pathogenesis of DSS-induced colitis ${ }^{25)}$. We therefore examined whether yuzu peel suppresses the activation of the NF- $\mathrm{B}$ pathway by using SEAP-expressing RAW 264.7 cells (RawBlue cells) in which SEAP reporter is expressed under the control of NF- $\kappa$ B response elements ${ }^{26)}$. RAW 264.7 cells were simultaneously treated with $50 \mathrm{ng} / \mathrm{mL}$ of LPS and different concentrations of RPMI 1640 extract of yuzu peel, and SEAP protein secreted into the culture medium was measured after incubation for $24 \mathrm{~h}$. As shown in Fig. 3A, yuzu peel extract significantly suppressed LPS-induced SEAP production, demonstrating that the extract potentially suppressed the activation of the NF- $\mathrm{BB}$ pathway.
To further confirm whether yuzu peel extract suppresses endogenous NF- $\kappa$ B pathway activation, we performed immunohistochemical analysis of p65 protein, a major subunit of $N F-\kappa B$. NF- $\kappa$ B exists in an inactive form in the cytoplasm owing to its interaction with $\mathrm{I} \kappa \mathrm{B} \alpha$, an inhibitor of $\mathrm{NF}-\kappa \mathrm{B}$. After activation of $\mathrm{I} \kappa \mathrm{B}$ kinase by stimulation, including LPS and TNF- $\alpha$, I $\mathrm{B} \alpha$ is phosphorylated, ubiquitinated, and degraded by proteasomes. The dissociated NF- $\mathrm{B}$ becomes free to translocate to the nucleus where it activates specific target genes through selective binding to the NF-кB response element. Through such mechanisms, $\mathrm{NF}-\kappa \mathrm{B}$ regulates the expression of a wide variety of genes, including TNF- $\alpha$. Thus, the activation of the NF- $\kappa \mathrm{B}$ pathway is characterized by the translocation of p65 from the cytoplasm to the nucleus.

To examine the subcellular localization of p65, RAW 264.7 cells were pretreated with RPMI 1640 extract of yuzu peel for $24 \mathrm{~h}$, and then treated with LPS for $30 \mathrm{~min}$. As show in Fig. 3B, whereas p65 was localized in the cytosol in unstimulated cells (upper panel), it was translocated to the nucleus by LPS stimulation (middle panel). As expected, p65 partially remained in the cytoplasm by the pretreatment with yuzu peel extract(Fig. 3B, bottom panel). Quantitative analysis demonstrated that the LPS-induced translocation of p65 from the cytoplasm to the nucleus was suppressed by yuzu peel extract (Fig. 3C), indicating that yuzu peel extract suppresses the activation of the endoge-

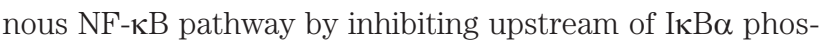
phorylation.

Hesperidin, which is present in yuzu peel extract in a large amount, has been reported to exert an inhibitory effect on NF-кB-mediated inflammatory responses ${ }^{27)}$. Naringin also has been reported to inhibit LPS-induced endotoxin shock in mice by suppressing NF- $\mathrm{B}$ pathway activation $^{28)}$. In addition, neohesperidin has been found to inhibit receptor activator of NF- $\mathrm{KB}$ ligand (RANKL) -induced activation $^{29)}$. Thus, although we cannot identify all the yuzu peel components involved in $\mathrm{NF}-\kappa \mathrm{B}$ suppression, it may be reasonable to assume that flavonoids, including hesperidin and naringin, play an important role in the suppression of $\mathrm{NF}-\kappa \mathrm{B}$ pathway activation.

\subsection{Antioxidant effect of yuzu peel against lipid}

Finally, we investigated the antioxidant effect of yuzu peel based on its radical scavenging activity. Antioxidant effect was examined by using the method for monitoring methyl linoleate oxidation, as reported previously ${ }^{20)}$. The same concentrations of ascorbic acid and hesperidin in PBS extract of yuzu peel were tested as controls, because we have identified the large amounts of hesperidin and ascorbic acid in yuzu peel extract. As shown in Fig. 4, ascorbic acid showed radical scavenging activity at the early stage of oxidation, whereas hesperidin exhibited radical scavenging activity at the later stage. The reason 


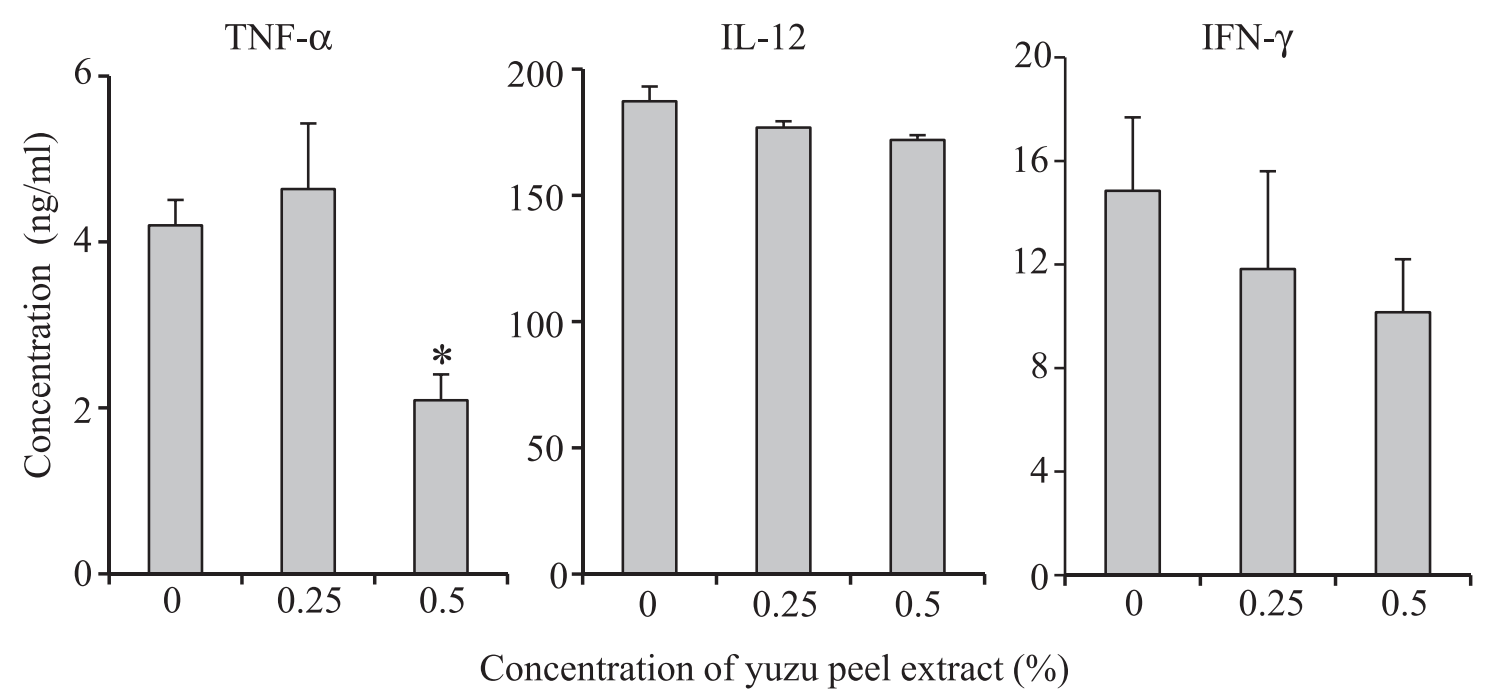

B

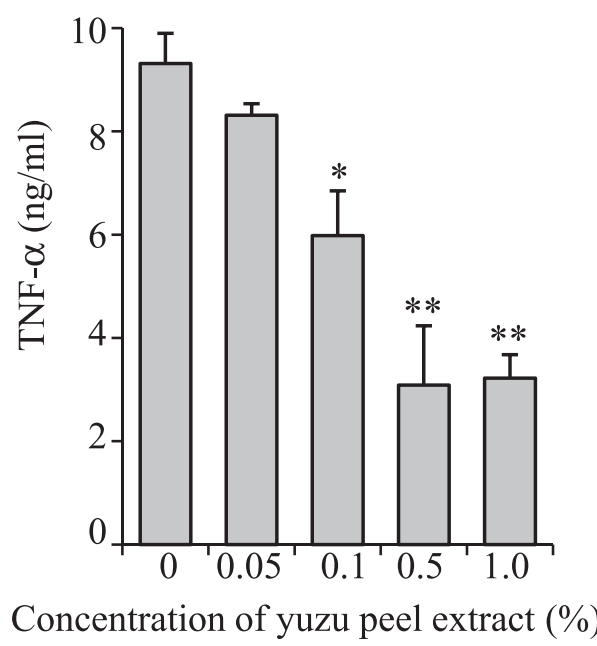

Fig. 2 Effects of yuzu peel extract on proinflammatory cytokine production in LPS-stimulated mice and RAW 264.7 cells. (A) Mice were intraperitoneally injected with $13 \mu \mathrm{g}$ of LPS alone or $0.25 \%$ or $0.5 \%$ PBS extract of yuzu peel and $13 \mu \mathrm{g}$ of LPS. Proinflammatory cytokine levels in plasma were assessed by ELISA. Data are expressed as means \pm SE of five animals in each group. $* p<0.05$ for comparison of $0 \%$ with each concentration of yuzu peel extract. (B) RAW264.7 cells were cultured with RPMI 1640 extract of yuzu peel and $250 \mathrm{ng} / \mathrm{mL}$ of LPS for $24 \mathrm{~h}$. Experiments were performed in triplicate and data are expressed as means \pm SE. $* p<0.05, * * p<0.01$ for comparison of $0 \%$ with each concentration of yuzu peel extract. 
A

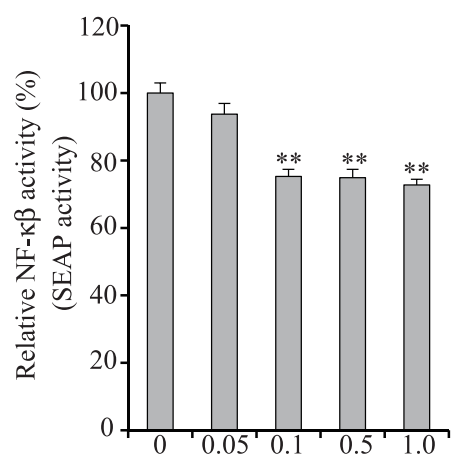

Concentration of yuzu peel extract $(\%)$
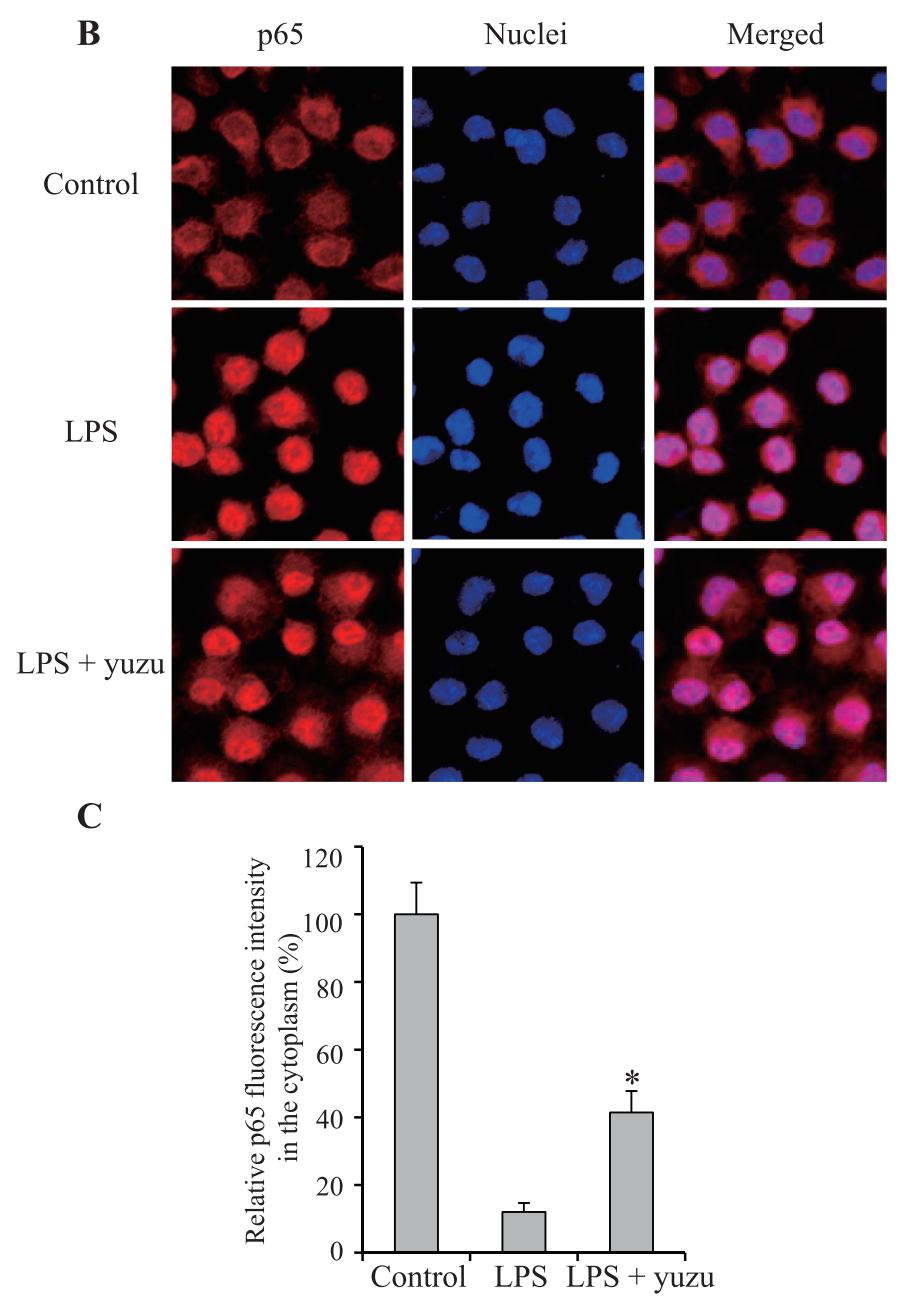

Fig. 3 Effects of yuzu peel extract on NF-кB activation in RAW 264.7 cells. (A) SEAP reporter assay using RAW 264.7 cells. Cells (Raw-Blue cells) were cultured with $50 \mathrm{ng} / \mathrm{mL}$ of LPS in the presence or absence of RPMI 1640 extract of yuzu peel for $24 \mathrm{~h}$, and then SEAP activity in culture medium was measured. Experiments were performed in four replicates, and data are expressed as means $\pm \mathrm{SE}$. ** $p<0.01$ for comparison of $0 \%$ with each concentration of yuzu peel extract. SEAP activity in the absence of yuzu peel extract was set at 100\%. (B) Representative fluorescence images of intracellular localization of p65 protein. p65 localization was examined under a confocal laser scanning microscope with indirect immunofluorescence using NF- $\mathrm{kB}$ p65 rabbit polyclonal antibody. Cells were stained with TO-PRO-3 to visualize nuclei. (C) Relative p65 fluorescence intensity in cytoplasm. Data are expressed as means \pm SE. $* p<0.05$ for comparison of LPS-alone-treated group with LPS + yuzu group. p65 fluorescence intensity in cytoplasm of unstimulated cells was set at $100 \%$. 
for this is unknown; nevertheless, we speculate that the oxidation products of hesperidin generated by radicals may be also effective against the next-coming radicals formed at the later stage. It is interesting that yuzu peel extract, whose hesperidin and ascorbic acid concentrations are the same as those of hesperidin and ascorbic acid positive controls, inhibited the oxidation much more efficiently than those controls. This may be attributed to the fact that antioxidative compounds other than ascorbic acid and hesperidin are present in the extract and flavonoids other than hesperidin contributed synergistically to the total antioxidant effect ${ }^{30)}$. It is therefore concluded that yuzu peel extract possesses moderate antioxidative effect against radicals and its effect is stronger than that of ascorbic acid and hesperidin at the same concentration.

\section{Conclusion}

We demonstrated that yuzu peel extract has an attenuating effect on DSS-induced symptoms, including weight loss, colon shortening, diarrhea, and visible fecal blood. We confirmed that yuzu peel extract suppressed LPS-stimulated TNF- $\alpha$ production(Fig. 2) and showed radical scavenging activity (Fig. 4), demonstrating that yuzu peel possesses both anti-inflammatory and antioxidant effects. Thus, it may be reasonable to assume that yuzu peel attenuated DSS-induced colitis symptoms due to the coordination

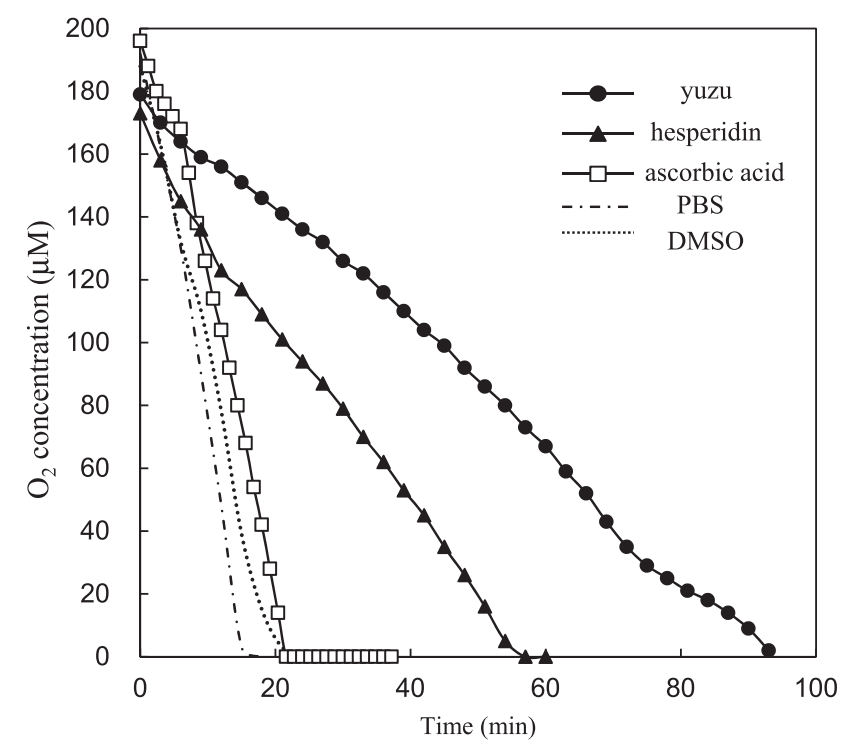

Fig. 4 Antioxidant activity of yuzu peel extract. PBS extract of yuzu peel was used at 250-fold dilution with PBS. As positive control, hesperidin and ascorbic acid were dissolved in DMSO and PBS, respectively, to make the same concentrations as those of hesperidin and ascorbic acid in the extract. between anti-inflammatory effect and antioxidant effect against lipids in vivo.

\section{Conflicts of Interest}

A part of this study was funded by Shikoku Research Institute Inc. for about three years from 2010 to 2012. Mika Ishioka was an employee of Shikoku Research Institute Inc.

\section{Supporting information}

The material is available free of charge via the Internet at http://dx.doi.org/10.5650/jos.ess 17184

Fig. S1: HPLC chart of yuzu peel extract (A) and (B-G) MS and MS/MS spectra for peaks 1(B), 2(C), 3(D), 4(E), $5(\mathrm{~F})$, and $6(\mathrm{G})$ in A. Fig. S2: Cytokine levels in supernatant of colon tissue culture. Fig S3: The plasma levels of tHETE in DSS-treated mice fed yuzu peel or the standard diet. Fig. S4: Effects of yuzu peel extract on viability.

\section{References}

1) Sanchez-Fidalgo, S.; Sanchez de Ibarguen, L.; Cardeno, A.; Alarcon de la Lastra, C. Influence of extra virgin olive oil diet enriched with hydroxytyrosol in a chronic DSS colitis model. Eur. J. Nutr. 51, 497-506 (2012).

2) Hanai H.; Iida T.; Takeuchi K.; Watanabe F.; Maruyama Y.; Kageoka M.; Ikeya K.; Yamada M.; Kikuyama M.; Iwaoka Y.; Hirayama K.; Nagata S.; Sato Y.; Hosoda Y. Intensive granulocyte and monocyte adsorption versus intravenous prednisolone in patients with severe ulcerative colitis: an unblinded randomised multi-centre controlled study. Dig. Liver Dis. 40, 433-40 (2008).

3) Oz, H.S.; Chen, T.S.; McClain, C.J.; de Villiers, W.J. Antioxidants as novel therapy in a murine model of colitis. J. Nutr. Biochem. 16, 297-304(2005).

4) Yarilina, A.; Xu, K.; Chen, J.; Ivashkiv, L.B. TNF activates calcium-nuclear factor of activated $\mathrm{T}$ cells (NFAT)c1 signaling pathways in human macrophages. Proc. Natl. Acad. Sci. USA 108, 1573-1578(2011).

5) Beutler, B.; Brown, T. A CAT reporter construct allows ultrasensitive estimation of TNF synthesis, and suggests that the TNF gene has been silenced in nonmacrophage cell lines. J. Clin. Invest. 87, 1336-1344 (1991).

6) Reimund, J.M.; Wittersheim, C.; Dumont, S.; Muller, C.D.; Baumann, R.; Poindron, P.; Duclos, B. Mucosal inflammatory cytokine production by intestinal biopsies in patients with ulcerative colitis and Crohn's disease. J. Clin. Immunol. 16, 144-150 (1996). 


\section{Yuzu Peel Attenuates DSS-induced Colitis}

7) Babbs, C.F. Oxygen radicals in ulcerative colitis. Free Radic. Biol. Med. 13, 169-181 (1992).

8) Petronilho, F.; Michels, M.; Danielski, L.G.; Goldim, M.P.; Florentino, D.; Vieira, A.; Mendonca, M.G.; Tournier, M.; Piacentini, B.; Giustina, A.D.; Leffa, D.D.; Pereira, G.W.; Pereira, V.D.; Rocha, J.B. Diphenyl diselenide attenuates oxidative stress and inflammatory parameters in ulcerative colitis: A comparison with ebselen. Pathol. Res. Pract. 212, 755-760 (2016).

9) Reddy, J.G.; Loftus, E.V.Jr. Safety of infliximab and other biologic agents in the inflammatory bowel diseases. Gastroenterol. Clin. North Am. 35, 837-855 (2006).

10） Miao, X.P.; Sun, X.N.; Cui, L.J.; Cao, Q.F.; Zhuang, G.F.; Deng, T.Z.; Zhang, D.Y. Suppressive effect of pectic polysaccharides extracted from Rauwolfia verticillata (Lour.) Baill.var.hainanensis Tsiang on inflammation by regulation of NF- kappa B pathway and interleukin-17 in mice with dextran sulphatesodium-induced ulcerative colitis. Asian Pac. J. Trop. Med. 8, 147-152 (2015).

11) Yu, H.Y.; Park, S.W.; Chung, I.M.; Jung, Y.S. Anti-platelet effects of yuzu extract and its component. Food Chem. Toxicol. 49, 3018-3024(2011).

12) Hirota, R.; Roger, N.N.; Nakamura, H.; Song, H.S.; Sawamura, M.; Suganuma, N. Anti-inflammatory effects of limonene from yuzu (Citrus junos Tanaka) essential oil on eosinophils. J. Food Sci. 75, H87-92 (2010).

13) Sawamura, M.; Wu, Y.; Fujiwara, C.; Urushibata, M. Inhibitory effect of yuzu essential oil on the formation of $\mathrm{N}$-nitrosodimethylamine in vegetables. J. Agric. Food Chem. 53, 4281-4287 (2005).

14）Yoo, K.M.; Lee, K.W.; Park, J.B.; Lee, H.J.; Hwang, I.K. Variation in major antioxidants and total antioxidant activity of Yuzu (Citrus junos Sieb ex Tanaka) during maturation and between cultivars. J. Agric. Food Chem. 52, 5907-5913(2004).

15) Kuraya, E.; Nakada, S.; Touyama, A.; Itoh, S. Improving the antioxidant functionality of Citrus junos Tanaka (yuzu) fruit juice by underwater shockwave pretreatment. Food Chem. 216, 123-129 (2017).

16) Yu, H.Y.; Ahn, J.H.; Park, S.W.; Jung, Y.S. Preventive effect of yuzu and hesperidin on left ventricular remodeling and dysfunction in rat permanent left anterior descending coronary artery occlusion model. PLOS One 10, e110596 (2015).

17) Kim, S.H.; Hur, H.J.; Yang, H.J.; Kim, H.J.; Kim, M.J.; Park, J.H.; Sung, M.J.; Kim, M.S.; Kwon, D.Y.; Hwang, J.T. Citrus junos Tanaka peel extract exerts antidiabetic effects via AMPK and PPAR-gamma both in vitro and in vivo in mice fed a high-fat diet. Evid. Based Complement. Alternat. Med. 2013, 921012 (2013).
18) Melgar, S.; Karlsson, A.; Michaelsson, E. Acute colitis induced by dextran sulfate sodium progresses to chronicity in C57BL/6 but not in BALB/c mice: correlation between symptoms and inflammation. Am. J. Physiol. Gastrointest. Liver Physiol. 288, G1328-1338(2005).

19) Tabei, Y.; Murotomi, K.; Umeno, A.; Horie, M.; Tsujino, Y.; Masutani, B.; Yoshida, Y.; Nakajima, Y. Antioxidant properties of 5-hydroxy-4-phenyl-butenolide via activation of Nrf2/ARE signaling pathway. Food Chem. Toxicol. 107, 129-137 (2017).

20) Umeno, A.; Takashima, M.; Murotomi, K.; Nakajima, Y.; Koike, T.; Matsuo, T.; Yoshida, Y. Radical-scavenging activity and antioxidative effects of olive leaf components oleuropein and hydroxytyrosol in comparison with homovanillic alcohol. J. Oleo Sci. 64, 793-800 (2015).

21) Xu, L.; Yang, Z.L.; Li, P.; Zhou, Y.Q. Modulating effect of Hesperidin on experimental murine colitis induced by dextran sulfate sodium. Phytomedicine 16, 989995 (2009).

22) Yoshida, Y.; Kodai, S.; Takemura, S.; Minamiyama, Y.; Niki, E. Simultaneous measurement of F2-isoprostane, hydroxyoctadecadienoic acid, hydroxyeicosatetraenoic acid, and hydroxycholesterols from physiological samples. Anal. Biochem. 379, 105-115(2008).

23) Tsuboi, Y.; Abe, H.; Nakagawa, R.; Oomizu, S.; Watanabe, K.; Nishi, N.; Nakamura, T.; Yamauchi, A.; Hirashima, M. Galectin-9 protects mice from the Shwartzman reaction by attracting prostaglandin E2producing polymorphonuclear leukocytes. Clin. Immunol. 124, 221-233 (2007).

24) Li, Q.; Verma, I.M. NF-kappaB regulation in the immune system. Nat. Rev. Immunol. 2, 725-734(2002).

25) Biasi, F.; Leonarduzzi, G.; Oteiza, P.I.; Poli, G. Inflammatory bowel disease: mechanisms, redox considerations, and therapeutic targets. Antioxid. Redox. Signal. 19, 1711-1747 (2013).

26) Kang, J.; Xie, C.; Li, Z.; Nagarajan, S.; Schauss, A.G.; $\mathrm{Wu}, \mathrm{T}$; $\mathrm{Wu}, \mathrm{X}$. Flavonoids from acai (Euterpe oleracea Mart.) pulp and their antioxidant and anti-inflammatory activities. Food Chem. 128, 152-157 (2011).

27) Saiprasad, G.; Chitra, P.; Manikandan, R.; Sudhandiran, G. Hesperidin alleviates oxidative stress and downregulates the expressions of proliferative and inflammatory markers in azoxymethane-induced experimental colon carcinogenesis in mice. Inflamm. Res. 62, 425440 (2013).

28) Kanno, S.; Shouji, A.; Tomizawa, A.; Hiura, T.; Osanai, Y.; Ujibe, M.; Obara, Y.; Nakahata, N.; Ishikawa, M. Inhibitory effect of naringin on lipopolysaccharide(LPS)induced endotoxin shock in mice and nitric oxide production in RAW 264.7 macrophages. Life Sci. 78, 673$681(2006)$.

29） Tan, Z.; Cheng, J.; Liu, Q.; Zhou, L.; Kenny, J.; Wang, T.; 
Lin, X.; Yuan, J.; Quinn, J.M.; Tickner, J.; Hong, G.; Qin, A.; Zhao, J.; Xu, J. Neohesperidin suppresses osteoclast differentiation, bone resorption and ovariectomised-induced osteoporosis in mice. Mol. Cell Endocrinol. 439, 369-378(2017).
30) Freeman, B.L.; Eggett, D.L.; Parker, T.L. Synergistic and antagonistic interactions of phenolic compounds found in navel oranges. J. Food Sci. 75, C570-576 (2010). 